\title{
Acyclic Coloring of Star Graph Families
}

\author{
Dr. K. Thilagavathi \\ Associate Professor \\ Department of mathematics \\ Kongunadu Arts and Science College Coimbatore \\ Tamilnadu-641029, India.
}

\author{
Mr. P. Shanas Babu \\ Research Scholar \\ Department of mathematics \\ Kongunadu Arts and Science College Coimbatore \\ Tamilnadu-641029, India.
}

\begin{abstract}
In this paper, we discuss the acyclic vertex colouring and acyclic chromatic number of middle graph, central graph and total graph of star graph.
\end{abstract}

\section{General Terms}

Middle graph, central graph and total graph of star graph are denoted by $M\left(K_{1, n}\right), C\left(K_{1, n}\right)$ and $T\left(K_{1, n}\right)$ respectively.

\section{Keywords}

Middle graph, central graph, total graph, acyclic colouring, acyclic chromatic number.

\section{INTRODUCTION}

Let $G$ be a graph with vertex set $V(G)$ and edge set $E(G)$. The middle graph [6,9] of $G$, denoted by $M(G)$ is defined as follows. The vertex set of $M(G)$ is $V(G) \cup E(G)$.Two vertices $x, y$ in the vertex set of $M(G)$ are adjacent in $M(G)$ in case one of following holds:

(i) $x, y$ Are in $E(G)$ and $x, y$ are adjacent in $G$. (ii) $x$ Is in $V(G), y$ is in $E(G)$, and $x, y$ are incident in $G$.

Let $G$ be a finite undirected graph with no loops and multiple edges. The central graph [16] of a graph $G, C(G)$ is obtained by subdividing each edge of $G$ exactly once and joining all the nonadjacent vertices of $G$. By the definition $p_{c G}=p+q$. For any

$p, q$ graph there exist exactly $p$ vertices of degree $p-1$ and $q$ vertices of degree 2 in $C(G)$.

Let $G$ be a graph with vertex set $V(G)$ and edge set $E(G)$. The total graph [6,9] of $G$, denoted by $T(G)$ is defined as follows. The vertex set of $T(G)$ is $V(G) \cup E(G)$.Two vertices $x, y$ in the vertex set of $T(G)$ are adjacent in $T(G)$ in case one of the following holds:

(i) $x, y$ are in $V(G)$ and $x$ is adjacent to $y$ in $G$.(ii) $x, y$ are in $E(G)$ and $x, y$ are adjacent in $G$. (ii) $x$ is in $V(G), y$ is in $E(G)$, and $x, y$ are incident in $G$.

A proper vertex colouring of a graph is acyclic if every cycle uses at least three colours [13].The acyclic chromatic number of $G$, denoted by $a G$, is the minimum $k$ such that $G$ admits an acyclic $k$-colouring.

\section{ACYCLIC COLOURING OF $M\left(K_{1, n}\right)$}

\subsection{Theorem}

For any star graph $K_{1, n}$ the acyclic chromatic number, $a\left[M\left(K_{1, n}\right)\right]=n+1$.

\section{Proof}

Consider the star graph $K_{1, n}$ with $V K_{1, n}=v_{1} v_{2}, v_{3}, \ldots v_{n}, v$ with $v$ as the root vertex. In middle graph $M\left(K_{1, n}\right)$, by the definition each edge $v v_{i}$ for $1 \leq i \leq n$ of $K_{1, n}$ is subdivided by the vertex $e_{i}$ in $M\left(K_{1, n}\right)$. and the vertices $e_{1}, e_{2}, e_{3}, \ldots e_{n,} v$ induce a clique of order $n+1$ in $M\left(K_{1, n}\right)$ i.e.,

$V\left(M\left(K_{1, n}\right)\right)=\left\{v_{i} / 1 \leq i \leq n\right\} \bigcup\left\{e_{i} / 1 \leq i \leq n\right\} \bigcup\{v\}$. Now assign a proper colouring to these vertices as follows. Consider a colour class $C=c_{1}, c_{2}, c_{3}, \ldots . c_{n+1}$. Assign the colour $c_{i}$ to the vertex $e_{i}$ for $i=1,2, . . n$ and the colour $c_{n+1}$ to the vertex $v$ and to $v_{i}$ for $i=1,2, . . n$. The colouring is minimum, as $M\left(K_{1, n}\right)$ contains a clique of order $n+1$, minimum $n+1$ colours are required for its proper colouring. Next we have to prove that the above said coloring is acyclic. It is obvious that to form a bichromatic cycle both colours should occur at least twice. But in the above said colouring, the only colour class which occur atleast twice is $c_{n+1}$ and all other colour classes occur only once in the colouring procedure. Hence the above said colouring is acyclic.

Thus $a\left[M\left(K_{1, n}\right)\right]=n+1$.

\section{Example}

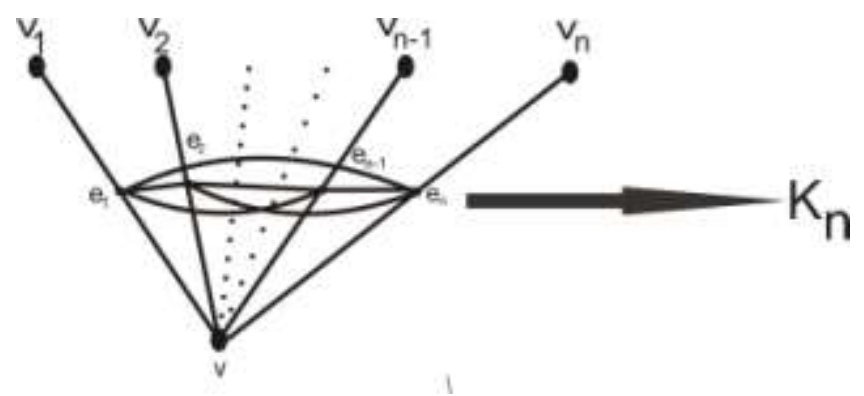

Figure 1 
$a\left[M\left(K_{1, n}\right)\right]=n+1$

\section{ACYCLIC COLOURING OF $C\left(K_{1, n}\right)$}

\subsection{Theorem}

For the graph $K_{1, n}$ the acyclic chromatic number $a\left[\begin{array}{ll}C & K_{1, n}\end{array}\right]=n, n \geq 3$.

\section{Proof}

Consider the

star

graph

$K_{1, n}$ with

$V K_{1, n}=u_{1} u_{2}, u_{3}, \cdots u_{n}, u_{0}$ with $u_{0}$ as the root vertex. In $C\left(K_{1, n}\right)$, let $u_{i, 0}$ represents the newly introduced vertex in the edge connecting $u_{i}$ and $u_{0}$ for $i=1,2 \ldots n$. Now assign a proper colouring to these vertices as follows. Consider a colour class $C=c_{1}, c_{2}, c_{3}, \ldots . c_{n}$. Assign the colour $c_{i}$ to the vertex $u_{i}$ for $i=1,2 \ldots n$ and the colour $c_{1}$ to the vertex $u_{0}$. Such a colouring excludes the newly introduced vertices. The colour $c_{2}$ is assigned to the newly introduced vertices $u_{i, 0}$ for $i \neq 2$ and the colour $c_{3}$ is assigned to $u_{2,0}$. The colouring is minimum, as $C\left(K_{1, n}\right)$ contains the subgraph $K_{n}$, minimum $n$ colours are required for its proper colouring. Next we have to prove that the above said coloring is acyclic. It is obvious that to form a bichromatic cycle both colours should occur at least twice. So in the above said colouring, the color classes $c_{k}, 4 \leq k \leq n$ never induce a 2-chromatic cycle (it occur only once in the colouring procedure). Now we need to examine the subgraphs induced by $\left\langle c_{i}, c_{j}\right\rangle$ for $i=1,2$ and $j=2,3$ with $i \neq j$ whether they induce a 2-chromatic cycle or not.

Case 1. If $i=1$ and $j=2$, then the sub graph induced by $\left\langle c_{1}, c_{2}\right\rangle$ is a tree with $n-3$ pendent vertices, which is clearly a forest.

Case 2. If $i=1$ and $j=3$, then the subgraph induced by $\left\langle c_{1}, c_{3}\right\rangle$ is the disjoint union of $P_{2}$ with itself. That is it forms a linear forest.

Case 3. If $j=2$ and $j=3$, then the subgraph induced by $\left\langle c_{2}, c_{3}\right\rangle$ is the union of a tree with $n-3$ isolated vertices, which also form a forest.

Thus any pair of the colour class will never induce a 2-chromatic cycle in the graph.

Therefore, $a\left[\begin{array}{ll}C & K_{1, n}\end{array}\right]=n, n \geq 3$.

Note: $a\left[\begin{array}{ll}C & K_{1, n}\end{array}\right]=3$, for $n=2$.

\section{Example}

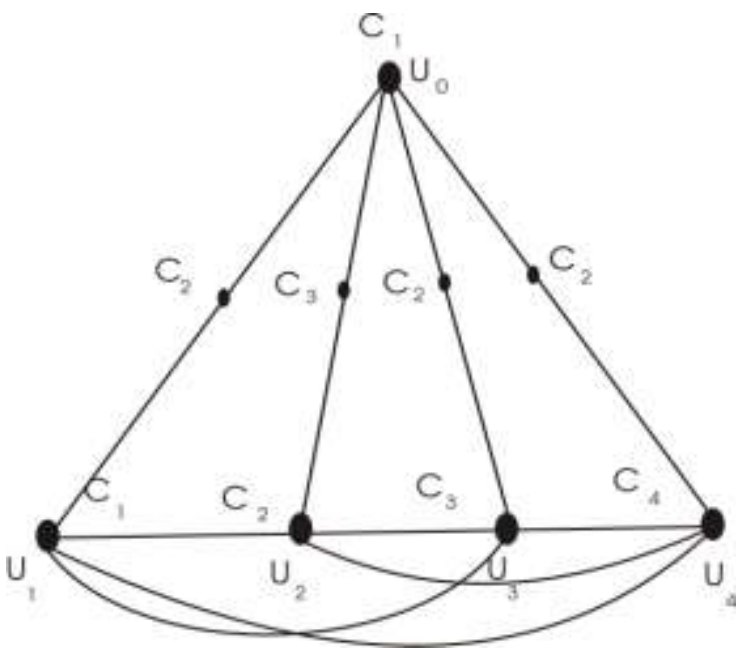

Figure 2

$a\left[\begin{array}{ll}C & K_{1,4}\end{array}\right]=4$

\section{ACYCLIC COLOURING OF $T\left(K_{1, n}\right)$}

\subsection{Theorem}

For any star graph $K_{1, n}$ the acyclic chromatic number, $a\left[T\left(K_{1, n}\right)\right]=n+1$.

\section{Proof}

Conside the star graph $K_{1, n}$ with $V K_{1, n}=v_{1}, v_{2}, v_{3} \ldots v_{n}, v$ with $v$ as the root vertex. . In Total graph $T\left(K_{1, n}\right)$, by the definition each edge $v v_{i}$ for $1 \leq i \leq n$ of $K_{1, n}$ is subdivided by the vertex $e_{i}$ in $T\left(K_{1, n}\right)$ and the vertices $e_{1}, e_{2}, e_{3}, \ldots e_{n}, v$ induce a clique of

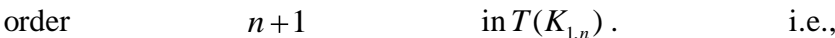
$V\left(M\left(K_{1, n}\right)\right)=\left\{v_{i} / 1 \leq i \leq n\right\} \bigcup\left\{e_{i} / 1 \leq i \leq n\right\} \bigcup\{v\}$. Now assign a proper colouring to these vertices as follows. Consider a colour class $C=c_{1}, c_{2}, c_{3}, \ldots . c_{n+1}$. Assign the colour $c_{i}$ to the vertex $e_{i}$ for $i=1,2, . . n$ and the colour $c_{n+1}$ to the vertex $v$. Now assign the colour $c_{1}$ to $v_{i}$ for $2 \leq i \leq n$ and $c_{2}$ to the remaining vertex $v_{1}$. The colouring is minimum, as $T\left(K_{1, n}\right)$ contains a clique of order $n+1$, minimum $n+1$ colours are required for its proper colouring. In the above said colouring, the color classes $c_{k}, 3 \leq k \leq n+1$ never induce a 2 -chromatic cycle (it occur only once in the colouring procedure). Also the subgraphs induced by $\left\langle c_{1}, c_{2}\right\rangle$ is the union of a path $P_{3}$ with $n-2$ isolated vertices, which is a forest. Thus any pair of the colour class will never induce a 2-chromatic cycle in the graph. That is the colouring is acyclic. 
Thus $a\left[T\left(K_{1, n}\right)\right]=n+1$.

\section{Example}

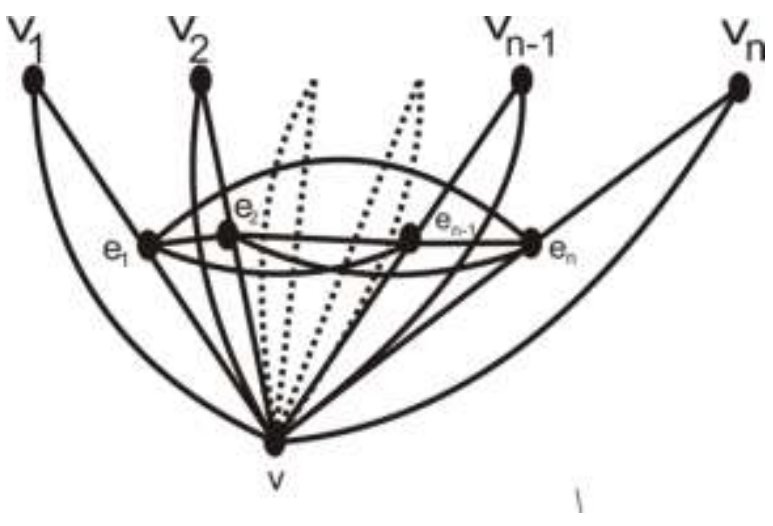

Figure 3

$$
a\left[T\left(K_{1, n}\right)\right]=n+1 .
$$

\section{REFERENCES}

[1] N. Alon, C. McDiarmid, and B. Reed. "Acyclic colourings of graphs". Random Structures and Algorithms, 2, 277288, 1990.

[2] J.A. Bondy and U.S.R. Murty, Graph theory with Applications . MacMillan, London, 976.

[3] O. V. Borodin "On acyclic colorings of planar graphs", Discrete Math. 25, 211-236, 1979.

[4] C. B. Boyer, A history of mathematics. New York, Wiley. 1968.

[5] A. E. Brouwer, A. M. Cohen and A. Neumaier, DistanceRegular Graphs. New York: Springer-Verlag, 1989.
[6] Danuta Michalak, On middle and total graphs with coarseness number equal 1, Spinger Verlag Graph Theory, Lagow (1981) proceedings, Berlin heidelberg, New York, Tokyo, pp. 139-150.

[7] Douglas B .West, Introduction To Graph Theory, Second Edition, Prentice-Hall of India Private Limited, New Delhi(2006).

[8] H. Eves, An Introduction to the History of Mathematics. New York: CBS College. 1983.

[9] Frank Harrary, Graph theory, Narosa Publishing House(2001).

[10] Frank Harary, Gray Chartrand, Ping Zhang, Geodetics sets in graphs. Discuss math. Graph Theory 20(2000)pp.129138.

[11] D. Frank Hsu, Harmonious Labelling of Windmill Graphs and Related Graphs, Journal of Graph Theory, Vol. 6 (1982), pp. 85-87.

[12] Graph Colouring, Wikipedia, the free Encyclopedia.

[13] B. Grünbaum. "Acyclic colorings of planar graphs". Israel J. Math., 14(3), 390-408, 1973.

[14] K. Thilagavathi, K.P. Thilagavathy and N. Roopesh, "The Achromatic colouring of graphs", Electronic notes in Discrete mathematics, 153-156, 33, 2009.

[15] K. Thilagavathi and Vernold Vivin.J, "Harmonious Colouring of Total graphs, n-Leaf, Central Graphs and circumdetic Graphs" PhD Thesis, Kongunadu Arts and Science college, Coimbatore.

[16] K.Thilagavathi and Vernold Vivin.J and Akbar Ali.M.M, "On Harmonious colouring of Central graphs" Advances and Appications in Discrete Mathematics, 2, 17-33, 2009.

[17] Vivin J. Vernold, M. Venkatachalam and Ali M.M. Akbar, "A note on achromatic coloring of star graph families" 23:3 (2009), 251-255. 